\title{
Aspectos motivacionales en la selección de carrera universitaria: Un Estado del Arte
}

\author{
Yulimer del Valle Uzcátegui
}

\begin{abstract}
Resumen: La selección de carrera universitaria es una de las acciones humanas más importantes para la vida individual y colectiva, se podría decir que es trascendental, pues se realiza, generalmente, en la adolescencia. Las razones para su elección provienen de aspectos motivacionales que pueden ser intrínsecos y/o extrínsecos, sin embargo, son pocas las investigaciones enfocadas a conocer las razones que promueven ese tipo de motivaciones. En esta investigación se busca comprender sobre los factores motivacionales que influyen en la selección de carrera, para esto se realizó un Estado del Arte, utilizando como base de datos el portal SciELO y tomando como concepto descriptivo central motivación, conectándolo con los términos: educación, selección de carrera, profesional, universidad y educación superior. Finalmente se encontraron dieciocho artículos, que fueron procesados mediante un Análisis de Contenido, encontrando cuatro categorías: 1 . Motivación para aprender, 2. La autodeterminación en la Motivación, 3. Motivación escolar y 4. Motivación en la selección de carrera universitaria. El estudio de estas categorías llevó a la conclusión que la motivación puede ser tanto intrínseca, como extrínseca, existiendo subniveles entre ellas, así mismo, que existe una escala de comprometimiento e interés por la realización de actividades, las cuales permiten pasar de una motivación extrínseca a una intrínseca. Además de que la motivación en un contexto académico depende de factores intervinientes, como son los sentimientos, las percepciones, las necesidades, las metas y el desempeño académico, como puntos fundamentales en la determinación de un comportamiento motivado.
\end{abstract}

Palabras claves: Motivación; Motivación Escolar; Selección de Carrera Universitaria.

\section{Aspectos motivacionais na escolha de cursos universitários: Um Estado da Arte}

Resumo: A escolha de um curso universitário é uma das ações humanas mais importantes na vida pessoal e social, pode-se dizer que é transcendental, dado que é feito, geralmente, na adolescência. As razões para a sua escolha vêm de aspectos motivacionais que podem ser intrínsecos e/ou extrínsecos, no entanto, são poucas as pesquisas focadas em conhecer as razões que promovem esse tipo de motivação. Nesta pesquisa, procura-se compreender sobre os fatores motivacionais que influenciam a escolha de cursos universitários, para isto, fez-se um Estado da Arte, utilizando, como base de dados, o portal SciELO e tendo, como conceito descritivo central, motivação, conectando-lhe com os termos: educação, seleção de carreira, profissional, universidade e educação superior. Finalmente, encontraram-se dezoito artigos, que foram tratados mediante uma Análise de Conteúdo, achando-se quatro categorias: 1. Motivação para aprender, 2. A autodeterminação na motivação, 3. Motivação escolar e 4. Motivação na escolha de cursos universitários. O estudo das categorias levou a concluir que a motivação pode ser tanto intrínseca como extrínseca, existindo sub- níveis entre elas, assim mesmo, existe uma escala de comprometimento e interesse pela realização das atividades, as quais permitem ir de uma motivação extrínseca a uma intrínseca. Ademais, a motivação no contexto acadêmico depende de fatores intervenientes, como são os sentimentos, as percepções, as necessidades, as metas e o desempenho acadêmico, como pontos fundamentais na determinação de um comportamento motivado.

Palabras-chaves: Motivação; Motivação Escolar; Escolha de cursos universitários.

\section{Motivational aspects in the selction of university course: A Stage of Art}

\begin{abstract}
The selection of university career is one of the most important human actions for individual and collective life, one could say that it is transcendental, because it is usually done in adolescence. The reasons for their choice come from motivational aspects that can be intrinsic and / or extrinsic, however, there are few researches focused on knowing the reasons that promote this type of motivations. This research seeks to understand the motivational factors that influence career selection, for this a State of the Art was made, using the SciELO portal as a database and taking as a central descriptive concept motivation, connecting it with the terms: education, career selection, professional,
\end{abstract}


university and higher education. Finally eighteen articles were found, which were processed through a Content Analysis, finding four categories: 1. Motivation to learn, 2. Selfdetermination in motivation, 3. School motivation and finally 4 . Motivation in the selection of university career. The study of these categories allowed us to conclude that the motivation can be both intrinsic, as extrinsic, existing levels between them, likewise, that there is a scale of commitment and interest for the realization of activities, which allow to go from an extrinsic motivation to an intrinsic In addition to that the motivation in an academic context depends on intervening factors, such as feelings, perceptions, needs, goals and academic performance, as fundamental points in the determination of a motivated behavior.

Keywords: Motivation; School Motivation; Choice of university courses.

\section{Introducción}

Escoger una carrera profesional es una decisión que parte de ideas y concepciones del pasado (lo que se desea ser), para realizar actividades en el presente (estudiar y formarse), con proyecciones hacia el futuro y cómo debería ser su desenvolvimiento profesional. Esto no es un evento sencillo, ya que es un proceso continuo que comienza desde el periodo escolar, donde hay acercamientos con diversas asignaturas y estas van dejando huellas, encaminando posibles selecciones. Después de seleccionar la carrera profesional, viene el periodo de prepararse, de estudiar, para ir al campo laboral y continuar con el desarrollo de aprendizajes a lo largo de la vida.

La elección de una carrera profesional es probablemente, según Skatova y Ferguson (2014), una de las primeras decisiones importantes de la vida de los jóvenes. Comprender los factores que afectan esta decisión es importante para proporcionar ayuda en la selección de carrera, considerando las expectativas de los estudiantes. Uno de esos factores es la motivación, la cual, para Cano (2008), es principalmente relevante en el nivel medio superior, etapa en la cual la carencia de motivación en los estudiantes origina apatía hacia las implicaciones personales, laborales y sociales de una carrera profesional.

Aunado a esto, PABLO-LERCHUNDI, NÚÑEZ-del-Río y GONZÁLEZ-TIRADOS (2015) indican que la motivación es un fenómeno que involucra procesos constantes de toma de decisiones y desencadena la iniciación, la elección y la persistencia en acciones que lleven a la culminación de una carrera profesional universitaria.

Para Ramírez y Cortes (2008), la motivación es toda acción humana asociada a un objetivo, meta o deseo, el cual se genera por un proceso homeostático, donde se activan estímulos, los cuales deben ser retroalimentados, produciendo respuestas multidimensionales de tipo fisiológica, psicológica e intelectual. Por tanto, se crean modificaciones de conducta en función de lo que perciben como bueno o malo, de acuerdo al juicio de valores de cada persona, llevando a una acción correspondiente y a la producción de una cadena emocional, 
que conlleva a la motivación,

Muchas otras investigaciones expresan como tipo de procesos motivacionales, los intrínsecos y los extrínsecos, expuestos por Deci (1971) y citado en los trabajos de MartinezHernandez y Valderrama-Juarez (2011) y Skatova y Ferguson (2014). Esta clasificación está relacionada a los intereses, deseos y expectativas que cada persona produce a partir de sus interacciones con el contexto, con los sentimientos, las creencias y los comportamientos observables. En base a esto, Skatova y Ferguson (2014) y Steimann, Bosch y Arasssa (2013) definen cada proceso de la siguiente manera:

Motivación Intrínseca: es aquella que es independiente del entorno donde la persona interactúa, está relacionada a los intereses individuales y solamente puede mudar por decisiones personales. Comúnmente está asociada a las metas, deseos, objetivos, proyectos y actividades que estimulan a hacer diferentes acciones para tener éxito en ellas.

Motivación extrínseca: relacionada a las situaciones del entorno de la persona y tiene influencia de acciones sociales, promueve la participación colectiva y sirve como un factor complementario para incentivar mudanzas en la motivación intrínseca.

En este sentido, Martínez y Valderrama (2011) indican que existen factores que influencian la continuidad de estudios universitarios, entre ellos, están la condición social, la estratificación, la movilidad social, el género, la educación de los padres, el lugar de origen, la trayectoria escolar, las características socio-económicas, los medios de comunicación, el apoyo de los padres o de una organización civil. Además de factores motivacionales que conducen la continuidad a la inversión profesional, los cuales promueven esfuerzos y habilidades en determinadas tareas, sean por interés propio, por deseo de ser independiente, de tener buenos salarios, de superación y/o por prestigio.

Sabiendo que la motivación está directamente relacionada a la selección y desenvolvimiento de una carrera profesional, es necesario investigar sus influencias. Por tal razón, se genera la siguiente pregunta de investigación: ¿Qué aspectos sócio-educacionales influyen en la motivación para la selección de formación profesional superior?

Para dar respuesta a esta pregunta, se plantea como objetivo de investigación identificar los aspectos motivacionales vinculados a la selección de formación profesional en curso superior.

\section{Motivación para Aprender}

La palabra motivación proveniente del latín movere y corresponde a todo aquello que 
se pueda mover. Esa acción de moverse, en términos de la teoría motivacional, es aquello que da como respuesta la acción de moverse (energizarse) y realizar actividades que lleven a un propósito en particular, un objetivo o una meta (direccionar) (PINTRICH, 2003). Es considerado como un acto individual, que depende de las experiencias, cultura y necesidades, las cuales iniciaran, direccionaran, intensificaran y generaran persistencia en la conducta, dirigida hacia ese objeto que se desea alcanzar o que incomoda y precisa ser atendido para modificarlo (BERNARDINO et al, 2018).

Autores como García Garduño y Organista (2006) consideran la motivación como la fuerza que energiza, dirige y mantiene el comportamiento humano en un constante accionar, generando ímpetu e inspiración en las actividades que se realizan. Definiendo, así mismo, la desmotivación como la ausencia de ímpetu e inspiración en la acción humana. Estos autores, fundamentados en estudios de Reeve, Ryan y Deci (2004), indican que la motivación puede evidenciarse en diferentes disposiciones sociales, donde se tendrá personas proactivas y comprometidas, otras pasivas y alineadas y otras activas y orientadas al crecimiento.

Estas conductas dependen del tipo de motivación, pudiendo estar presente la motivación extrínseca y motivación intrínseca, además de indicar que ellas coexisten y su intensidad dependerá de cada situación y personal. Teniendo que, según Bzuneck y Guimarães (2007), la desmotivación es definida como la ausencia de intención para moverse.

Desde otra perspectiva, para Beluce y Oliveira (2016), la motivación es un constructo interno complejo que direcciona, altera y mantiene las metas y preferencias particulares de cada persona. Por tales razones, es determinante para alcanzar el éxito personal y está asociada a una mejor calidad de aprendizaje escolar, donde los docentes necesitan buscar estrategias realmente eficaces para motivar al estudiante. Así mismo, Bzuneck y Guimarães (2007) apuntan que la ausencia o poca motivación traerá como consecuencia innumerables problemas, porque no tendrá interés en realizar tareas o actividades que contribuyan con su aprendizaje.

Continuando en el contexto educacional, Pintrich y De Groot (1990) indican que la motivación es una variable compleja determinada por las interacciones de componentes relacionados con el contexto de las clases, sentimientos y creencias de los estudiantes en relación a su propia motivación y los comportamientos que asumen en aula.

Como se puede observar, cada investigación direccionó la definición de motivación en función de los diferentes autores citados en los trabajos analizados, entre ellos Ryan \& Deci (2000, 2001), Reeve, Deci y Ryan (2004), Pintrich y De Groot (1990) y Bzuneck (2001, 2007). Sus enfoques se pudeden clasificar en dos grupos, el primero está relacionado a lo que 
promueve la motivación en las personas y como es el comportamiento humano; el segundo hace relación con la educación y como la motivación infiere en ese proceso. En este orden de ideas se explicará, a detalle, cada enfoque.

En el primer enfoque, la motivación es concebida como la fuerza interna o externa, producto de lo que se desea lograr, que energiza, direcciona, altera y mantiene las preferencias y metas. Para lograr esto, el comportamiento humano se acciona, mediante un constructo interno complejo, que genera ímpetu e inspiración, o la ausencia de estas que corresponde a la desmotivación. Estas acciones se puede observar en tres tipos de condiciones sociales, explicadas por Reeve, Ryan y Deci (2004), como: ser personas proactivas y comprometidos, ser pasivas y alineadas o ser personas activas ${ }^{1}$ y orientadas al crecimiento.

En el segundo enfoque, la motivación del estudiante es considerada en función del aprendizaje y enseñanza, ya que se centra en cuestionamientos sobre cómo y porque algunos estudiantes parecen aprender y prosperar en la escuela, mientras otras luchan para alcanzar sus metas académicas o sobre el desarrollo de clases innovadoras que motivan al estudiante a aprender. (BZUNECK Y GUIMARÃES, 2007; PINTRICH, 2003). En razón de esto, el profesor debe conocer lo que genera motivación en sus estudiantes y desarrollar estrategias didácticas que puedan ser eficaces para lograr mejoras en la calidad del aprendizaje escolar y el éxito educacional.

\section{La Autodeterminación en la motivación}

La autodeterminación es la propuesta de Ryan y Deci (2000) que permite hacer una interfaz entre motivación humana y el área educativa, ya que permite hacer mejoras en la motivación implicada en el trabajo, en las relaciones personales y en el proceso de enseñanza y aprendizaje. El término de Autodeterminación es explicado por Rufini, Bzuneck y Oliveira (2012), y ellos indican que se refiere al comportamiento intencional en el desenvolvimiento de las actividades, el mismo puede ser autónomo o controlado. En el caso del autónomo, la actividad se realiza por placer, teniendo todos los componentes de la autodeterminación, como es la presencia del locus interno, libertad psicológica y posibilidad de elección. Por otra parte, las controladas pueden presentar ganas de hacer la actividad, satisfacción y creatividad en el desenvolvimiento, pero depende de la petición de otros o la búsqueda de recompensas.

Para Reeve, Ryan y Deci (2004), la motivación puede ser intrínseca o autónoma, es

\footnotetext{
${ }^{1}$ Persona activa se refiere a aquellas personas abiertas a nuevas actividades, a cambios, son entusiastas, sin prejuicio ante nuevas experiencias, los retos intensifican su motivación. Suelen ser creativos, novedosos, generadores de ideas, participativo, competitivo, deseoso de aprender y de solucionar problemas.
} 
decir, surge de lo que la persona desea hacer intencionalmente para sentir satisfacción; puede ser extrínseca o controlada, la cual no es intencional y surge por medio de una regulación interior, generalmente basada en el logro de metas u objetivos que acarrean una recompensa; la ausencia de ambas implica una desmotivación, es decir, la ausencia de ganas para hacer algo.

Al hablar de motivación intrínseca (BZUNECK y GUIMARÃES, 2007) se tiene que es una tendencia natural para ejercitar las capacidades personales por medio de desafíos, todo esto es un proceso de búsqueda de la novedad en lo que resulta interesante conocer y genera satisfacción durante su ejecución.

La motivación intrínseca, según Bernardino et al (2018), se divide en tres subcategorias, las cuales son: a. para Vivencia Estímulos (MIVE), son las actividades que se desean realizar solo por conocer nuevas sensaciones y experiencias. b. por Realización (MIR), es hacer algo por placer, para su realización personal o para descubrir cosas nuevas. c. para Saber (MIS), asociadas a acciones que generan satisfacción por aprender y/o entender algo.

En cuanto a sus implicaciones académicas, la motivación intrínseca, según García Garduño y Organista (2006) y Steimann, Bosch y Aiassa (2013), propicia la participación libre y activa en las actividades de clases, con una tendencia inherente a realizar retos y novedades, a disponer de tiempo y espacio para extender y ejercitar las capacidades alcanzadas y generando una tendencia a explorar y aprender por placer. Específicamente en el estudio de carreras universitarias, según Bernardino et al (2018), ayuda a la retención y profundización de los aprendizajes, contribuyendo a la formación de profesionales con mayor autonomía, con un carácter más reflexivo y crítico, necesario para el desarrollo de actitudes útiles en la resolución de problemas; generando el éxito académico con menor esfuerzo.

Por otro lado, la motivación extrínseca, es todo actor externo que influye en la realización de actividades, por tal razón en esta categoría se encuentra todo el proceso formativo escolar. Para Rufini, Bzuneck y Oliveira (2012, p. 54), “[...] la preocupación de todo profesor debe ser por los mejores resultados en los aprendizajes de los alumnos, lo que ocurrirá en función del comprometimiento en las actividades [...]"; tal comprometimiento viene dado por la participación activa y la emoción atribuida en la realización de las actividades, dando un mayor peso motivacional al aprendizaje y haciendo que la regulación externa se aproxime a un comportamiento autodeterminado, es decir, acercando al estudiante a una motivación externa del tipo integral, la cual será explicada más adelante.

Figura 1. Esquema sobre los diferentes tipos de motivación extrínseca, visto por etapas de desenvolvimiento, basado en García Garduño y Organista (2006) y Bzuneck yGuimarães 


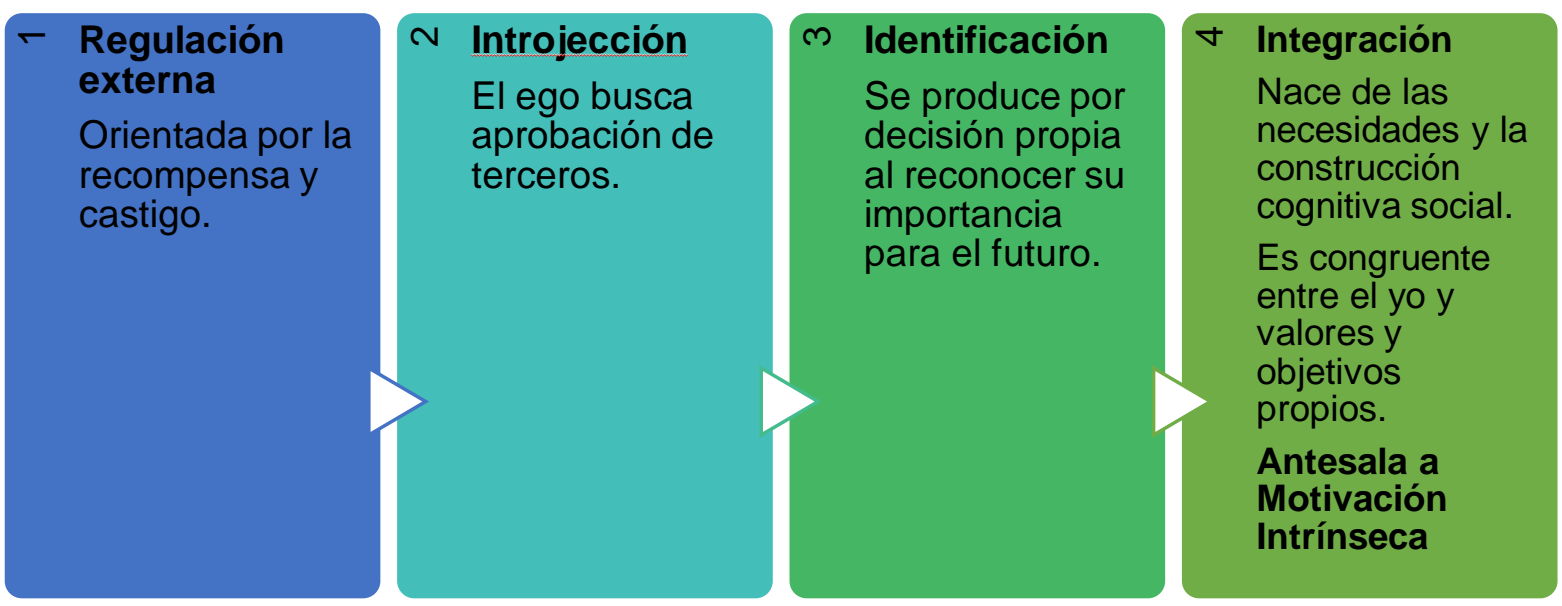

Según Pintrich (2003), consiste en cuatro niveles diferentes. El primero es de Control Externo (MEXE), se produce por presiones externas de alguien o algo, controlada por restricciones o recompensas. El segundo es por Introyección (MEI), consiste en una internalización de valores, pero aún se percibe un control extremo por personas, en la medida que busca ser aprobado por los otros.

El tercero es por Identificación (MEID), se refiere a la realización de una actividad y el reconocimiento de su importancia para acciones futuras, es más de control interno y autoadición de valores y objetivos. La última es llamada de Integración (MEIN), la cual nace de las necesidades y la construcción cognitiva social, refleja un alto control interno y es congruente entre el yo y los propios valores y objetivos. Para García Garduño y Organista (2006), Bzuneck y Guimarães (2007), la motivación extrínseca de Integración es considerada como la antesala a una Motivación Intrínseca, por su proximidad en el grado de autodeterminación, pero no coincide porque es sustentada en la realización de actividades para la obtención de metas y valores internalizados.

Este desenvolvimiento de actividades dentro de la motivación extrínseca hasta convertirse en antesala de lo que puede ser una motivación intrínseca se puede observar en la figura 1, en la cual se refleja cada motivación como una etapa, donde se deja de tener una conducta por premio o castigo, siendo cada vez más ligada a una acción, producto de los valores propios de cada persona, obteniendo una acción muy similar a una actitud antes las actividades de tipo de motivación intrínseca.

En el ámbito académico, según Avendaño y González (2012), esta puede influir negativamente, principalmente por el aumento de presión externa. A nivel universitario, se tiene que en muchas oportunidades las personas escogen sus carreras universitarias por motivos económicos, es decir, por los beneficios económicos que le puede generar; o por 
motivos familiares (bien sea por negocios, por tendencias o por metas no alcanzadas de los padres que desean ser logradas en sus hijos). Estas elecciones de carrera comúnmente generan personas menos comprometidos en sus estudios y profesión, ya que presentan ausencia de una motivación intrínseca, realmente ligada a sus valores, objetivos y necesidades personales.

Figura 2. Esquema sobre la Autodeterminación a partir de la información aportada por todos los artículos analizados en este apartado.

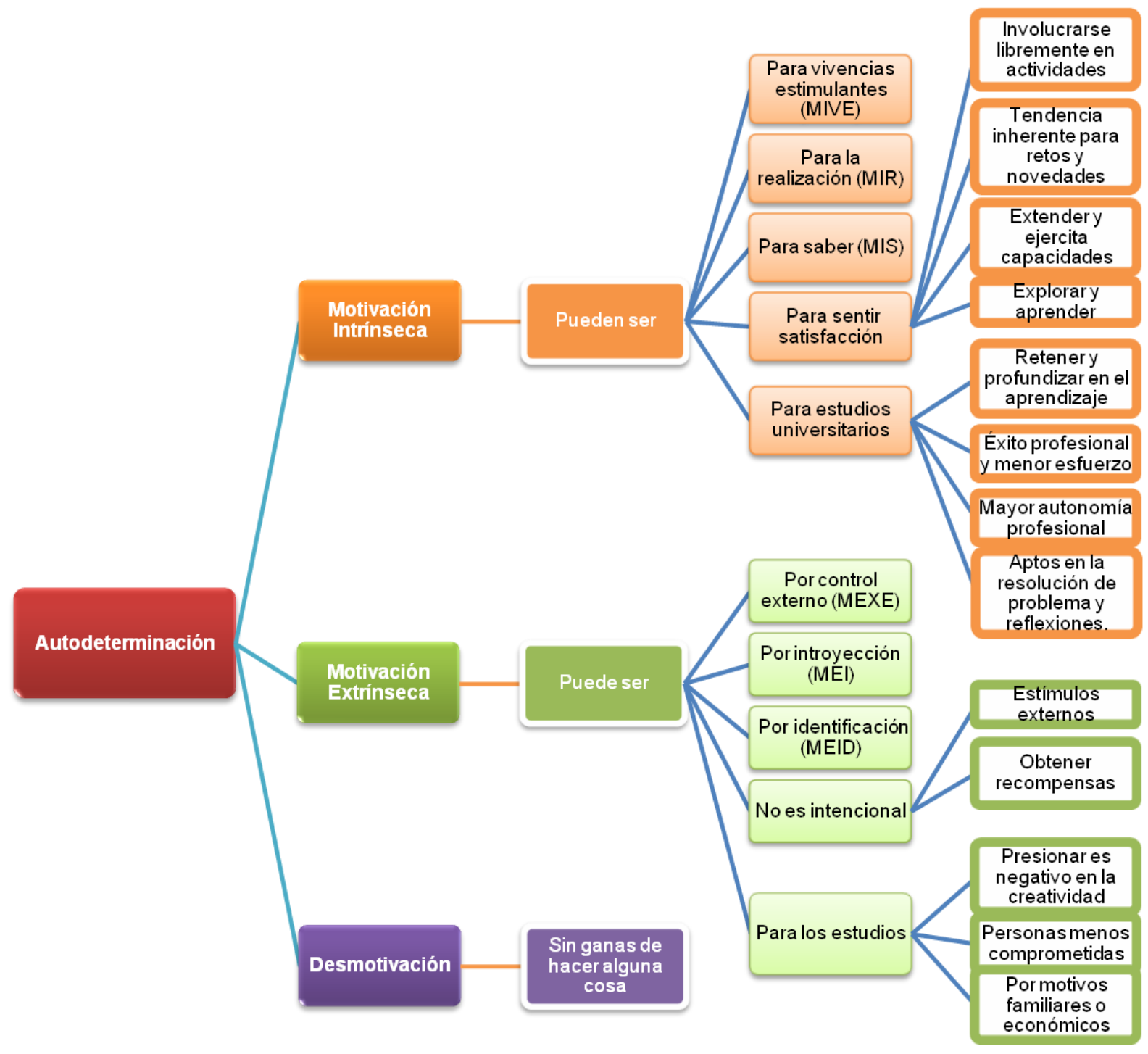

Todos los aportes presentados se complementan entre sí, tal como se observa en la figura 2. La autodeterminación expresa como se puede generar las acciones que se ejercen a lo largo del día, adjudicando a cada una de estas maneras unos posibles resultados de cómo se va a interactuar en cada actividad que se hace. Hay una motivación intrínseca, ligada a lo que se hace porque desea internamente, es eso que apasiona, interesa, a lo que se desea dedicar tiempo para realizar, esto es aplicable en los estudios y principalmente en las carreras 
profesionales.

Lo que genera ganas de invertir tiempo para aprender, para practicar, para mejorar, es aquello que moverá para ser personas activas ante esos eventos específicos. Esto corresponde a la motivación intrínseca y es independientemente de lo difícil que resulte ser el aprendizaje, esto genera personas comprometidas, por lo tanto, las carreras universitarias que se escogen a partir de la motivación intrínseca, generan personas capaces de generar cambios en la comunidad donde se desenvuelven.

Por otra parte, se tienen actividades que se realizan por presión externa, bien sea por alcanzar un objetivo concreto, realizar una tarea, aprobar una materia obligatoria, o cualquier otro tipo de recompensa. Cuando la carrera universitaria se escoge por motivos extrínsecos, se tienen personas menos comprometidas, a las cuales aprender les genera un esfuerzo mayor, no hay disposición ni compromiso, generando profesionales (en el caso que culminen los estudios) que harán el trabajo por hacerlo.

\section{Motivación Escolar}

Para definir la motivación escolar, se debe comprender lo complejo y amplio del tema. En este sentido, Steimann, Bosh y Aiassa (2013) apuntan que depende de una interrelación entre diferentes componentes, como son los cognitivos (asociado a la capacidad para aprender), los afectivos (relacionado a las emociones presentes en las diferentes circunstancias que puede envolver cada individuo), y el contexto social y cultural en el que se desenvuelve los estudiantes.

A modo de desglosar dichos aspectos, se tiene que sobre la motivación escolar influye: la disposición a aprender, los intereses intrínsecos, el valor personal atribuido a estar en clases, las emociones envueltas en la dinámica de la clase, la familia (la cual debe generar un ambiente propicio para un desarrollo educativo exitoso), la relación con los amigos, las situaciones propias de su relación con el entorno, su cultura, la relación entre pares, la relación profesor-estudiante, entre otros factores que pueden modificar la dinámica escolar (PINTRICH, 2003).

Ahondando un poco sobre la motivación del estudiante, Steimann, Bosch y Aiassa (2013) indican que debe existir una voluntad para aprender y que esta se relaciona con experiencias subjetivas, que le darán valor y relevancia a la acción de estudiar o hacer actividades académicas, modificando la disposición del estudiante en su accionar. Así como también se resalta que la motivación va a responder a situaciones externas, condiciones 
físicas, percepciones o representaciones mentales de la situación.

Por otra parte, Valenzuela et al (2015) conceptualizan la motivación escolar como la activación de recursos cognitivos para aprender, generando un valor a la tarea como un recurso que promueve un sentido de competencia, es decir, da apertura a un contacto directo con el contenido que se esté desarrollando, a un intercambio de ideas, a la profundización del contenido, al monitoreo, control y modificación del conocimiento que se está generando. De esta manera, según este autor, se dan motivos para aprender, ya que se tendría una aplicación del conocimiento y por onde la contextualización de los contenidos desarrollados.

Sin embargo, resulta importante conocer las ideas principales presentadas en los abordajes teóricos debatidos en los diversos artículos analizados, a fin de vislumbrar una comprensión de lo que ocurren en el entorno escolar. Esas teorías son las siguientes.

Autodeterminación, expuesta por Deci y Ryan (2000, 2001 y 2004) y ampliamente utilizada para explicar los tipos de motivación presentes en nuestro accionar, dando la opción de intrínseca, extrínseca y desmotivación. Como se explicó ampliamente en el apartado anterior. No obstante, en base a la autodeterminación surgió la autorregulación, la cual tiene como principal exponente Pintrich $(1990,1994,2001,2003)$ y también es ampliamente citado en el contexto de la motivación escolar, ya que está evocada a la explicación de los factores que influyen en la motivación de los estudiantes.

Autorregulación en el aprendizaje, para Pintrich y De Groot (1990), tiene tres componentes principales: el primero es incluir estrategias de metacognición ${ }^{2}$ para los estudiantes y tener una planificación, monitoreo y modificación de su cognición. El segundo corresponde a la gestión y control de los estudiantes en tareas académicas dentro del aula de clases, evitando distracciones para mantener el desarrollo de la actividad a cabalidad.

El tercero es la inclusión de estrategias cognitivas ${ }^{3}$ para que los estudiantes aprendan, recuerden y entiendan el contenido. Es la relevancia de recalcar que los tres componentes están conectados a la motivación individual de cada estudiante.

Modelo de Expectación/Valor, propuesto por Eccles y Wigfield (1995), ellos explican que la motivación al logro de una tarea es atribuido a la expectativa y el valor dado a esa tarea. En él se involucran dos aspectos fundamentales que Valenzuela et al (2015) explican con detenimiento, estos son expectativa y valor, referente a cada uno se tiene lo

\footnotetext{
${ }^{2}$ Las estrategias de metacognición corresponden a las que se refieren al conocimiento del propio conocimiento, implica la capacidad de evaluar, organizar y regular el conocimiento adquirido, supone la capacidad de entender cuáles son las competencias necesarias para resolver problemas. (Tonelotto, 2012).

${ }^{3}$ Las estrategias cognitivas son aquellas presentes durante la ejecución de una tarea y ayudan al aprendiz a asimilar las informaciones. Operan directamente en la información y su uso se refleja en la forma como el estudiante almacena, organiza y elabora la información. (Góes y Alliprandini, 2014).
} 
siguiente:

a) La expectativa viene dada por el poder realizar adecuadamente una determinada tarea, centrándose en competencias futuras, mientras que el constructo se localiza en el presente.

b) El valor es producto de cuatro componentes, el interés (disfrute o gusto por hacer la tarea), la importancia (depende de cuán importante es hacer adecuadamente la tarea), ambas ligadas a la teoría de Deci y Ryan (2000) apud Valenzuela et al (2015, p. 354) sobre la motivación intrínseca. Otro componente involucrado en estos dos aspectos es la utilidad (se refiere a la utilidad que tendrá la misma en el futuro) y el costo (es la percepción de cuanto tendrá que sacrificar o invertir para realizar la tarea).

c) Un tercer aspecto, agregado por Pintrich y De Groot (1990), corresponde a las reacciones emocionales involucradas en la tarea, en función de que ellas pueden generar diferentes reacciones como rabia, orgullo, culpa y entre otras como ansiedad, siendo esta última la más resaltante, ya que está asociada a perspectivas de competencia, teniendo que entre más ansiosos resulten, más difícil será realizar la tarea, además de la ausencia de estrategias cognitivas apropiadas en la ejecución de sus actividades.

Motivos que dan sentido a aprender, basados en la perspectiva de Valenzuela (2015), motivan a los estudiantes a aprender por: responsabilidad social, desarrollo personal, movilidad social, sobrevivencia y lo que denomina como "el mal menor".

Abordaje socio-cognitivista, el cual es descrito por Paiva y Boruchovitch (2012), y basado en el modelo de Deci y Ryan (2000), da una visión sobre la influencia de la motivación intrínseca y extrínseca en la escuela, teniendo la siguiente descripción:

Intrínseca: es la tendencia natural para buscar novedades e desafíos, las actividades son hechas por sus propias causas, es decir, porque le considera interesante o le genera satisfacción. Es de autonomía del estudiante y depende de su autorregulación en el aprendizaje. En el contexto escolar, las personas con este tipo de motivación se envuelven en actividades por la oportunidad para apropiarse de sus conocimientos y habilidades.

Extrínseca: es una motivación para trabajar en respuesta a algo externo, a tareas, por la recompensa externa, su finalidad es atender solicitaciones o presiones de otras personas o para demostrar competencias y habilidades.

En función de estas teorías, el proceso de enseñanza no se puede limitar a un grupo de estrategias, igualmente el aprendizaje no puede basarse en una herramienta de enseñanza, se requiere del entendimiento de la complejidad del ser humano para obtener mejores resultados académicos, tanto desde la perspectiva del docente, como del estudiante y de la comunidad 
que observa y espera el éxito para un bien común.

\section{Motivación en la selección de carrera profesional}

Escoger una carrera universitaria es una toma de decisiones, en la mayoría de los casos, irreversible, que corresponde hacia una meta de área de trabajo, ocupación o profesión (BERNARDINO et al, 2018). Así mismo, Montalvo-Aguilar et al (2013) indican que la motivación como fuerza motriz incide en todas las acciones del ser humano, por lo tanto afecta directamente la elección de una profesión y será la responsable de la disposición que tenga la persona de iniciar y continuar una carrera profesional independientemente de los sacrificios, infortunios, perspectivas desalentadoras, entre otros factores adversos; fundamentado en los ideales de lo que desea alcanzar.

La motivación para estudiar, según Paiva y Boruchovitch (2012), está ampliamente relacionada a la motivación escolar, pero especialmente con la motivación en la elección profesional, porque da origen y valoración a las metas a futuro, la tarea pasa a ser vista como instrumento para la consecución de metas. Continuando con los aportes de Eccle y Wigfiel (1995), el valor subjetivo de una tarea dada engloba cuatro componentes: el interés intrínseco, la utilidad, la importancia y el costo, teniendo diferentes relevancias en estos componentes, en función del valor de la propia meta en sí.

En este sentido, Bzuneck y Guimarães (2007) indican que la motivación escolar es afectada por la percepción de instrumentabilidad, es decir, en la medida que comprende la aplicación de ese conocimiento. Cuando un estudiante tiene una alta percepción de instrumentabilidad, y hace lo mejor posible las actividades de clases, esto conduce a una preparación al futuro, justamente con una actitud positiva hacia el futuro. Esto lleva a una mayor motivación y a mejores resultados académicos.

Tal como la motivación escolar, la motivación en la elección profesional también es compleja y dependerá de las teorías expuestas en motivación escolar. En este sentido, se presentan los diferentes aspectos que influyen en esta toma de decisiones, según las teorías estudiadas en el apartado anterior.

Según el modelo de Autodeterminación, expuesto por Reeve, Deci y Ryan (2004), se tienen aspectos tanto intrínsecos como extrínsecos involucrados en la elección de carrera. En cuanto a la motivación intrínseca, esta se fundamenta en una autorealización (satisfacción propia); en la que influyen aspectos vocacionales, es decir, aquellas actividades ligadas a una 
profesión, que dan satisfacción realizar; presenta motivos altruistas ${ }^{4}$. Aunque este último, para Said-Hung, Gratacós y Cobos (2017), puede ser considerado como un tipo de motivación específica que influye al tomar la decisión.

La motivación extrínseca, por su parte, presenta aspectos como la influencia familiar (como presión de los padres), recomendaciones, antiguos docentes, movilidad social, incentivos externos (asignaciones, posibilidades de estudio, becas), económicos. Estos factores se reflejan en no tener claro lo que se desea estudiar, selecciona algo en lo que no quiere trabajar, cambios de carrera, deseos por satisfacer necesidades principalmente económicas.

En relación a esto, Bzuneck y Guimarães (2007) sugieren que las formas de motivación autodeterminadas, así como la propia motivación intrínseca, dependen positivamente del comportamiento interpersonal caracterizadas como promotores de autonomía.

Por otra parte, Pereira et al (2017) exponen cuatro (4) componentes principales relacionados a la motivación en el contexto universitario. El primero es el contexto sociocultura, en el cual intervienen las actitudes, creencias y comportamientos de partida. El segundo es el ambiente del salón de clases, donde coexisten una interacción con los tipos de tarea, comportamiento del docente y los métodos instruccionales. El tercero son los factores internos de cada persona y el último corresponde al comportamiento motivado del modelo de Pintrich (1994), donde se consideran los sentimientos, las percepciones, las necesidades, las metas y el desempeño académico como puntos fundamentales en la determinación de un comportamiento motivado. El modelo permite identificar la motivación presente en función de los comportamientos observados.

Basados en Bernardino et al (2018) y Avendaño y González (2012), en la selección de carrera influye la personalidad, el entorno donde se desenvuelve (familia, modelos de referencia, tendencia social) y los deseos de satisfacer necesidades. Otro factor, expuesto por Avendaño y González (2012) y Montalvo-Aguilar et al (2013), corresponde a la influencia propia del género, destacando que las mujeres realizan selección orientadas a carreras sociales y ligadas a las humanidades, por motivos netamente intrínsecos. Mientras que los hombres se basan en motivos instrumentales, como proyección de salario y aspectos científicos y/o técnicos.

Aunado a esto, Watt y Richardson (2007) consideran tres tipos de motivación: la intrínseca, la extrínseca y la altruista (relacionada a la búsqueda de mejoras para la sociedad),

\footnotetext{
${ }^{4}$ Las personas altruistas, son aquellas que están a disposición de ayudar a otros, trabajar al servicio de los demás, en procura de un bienestar colectivo, comúnmente asociado a carreras como medicina, enfermería y educadores.
} 
proponiendo la escala FIT-choice (Factors Influencing Teaching choice), principalmente basado en la teoría expectativa-valor diseñada Eccles y Wigfield (1995). Dicha escala describe cuatro (4) elementos que van a influir en la selección de carrera, esos son:

a) Auto percepción: habilidad para explicar lo que se sabe.

b) Valor de la tarea, que está orientado a una utilidad personal (seguridad, movilidad laboral, conciliación familiar) o a una utilidad social (contribución social, mejoras en la sociedad).

c) Carrera de segunda opción, correspondiente a una selección por descarte.

d) Expectativas y creencias, relacionado con la exigencia de la carrera y de la profesión al momento de ejercer.

Desde la perspectiva de Bravo, González y Maytorena (apud ROMAN, 2013 p. 163), el éxito académico en la carrera que se escoja dependerá del interés que tenga el estudiante por la tarea, por los exámenes, por la clase del profesor y por la capacidad y esfuerzo que esté dispuesto a ejercer.

Específicamente en el ámbito de la profesión docente, Avendaño y González (2012) exponen que entre las motivaciones para la selección destaca el querer trabajar con niños y/o jóvenes, gusto por enseñar, gusto por la especialidad, gusto por la educación en sí y su importancia social.

\section{Abordaje Metodológico}

El desenvolvimiento de esta investigación es de naturaleza teórica, bibliográfica, de tipo Estado del Arte que, según Ferreira (2002), es una metodología que busca mapear y discutir una determinada producción académica en diferentes campos del conocimiento. En este sentido, se delimita la producción de datos de la investigación en base a los artículos encontrados en Scientific Electronic Librery Online (SciELO), por considerar la cantidad de revistas digitales asociadas y de acceso libre. Para la organización del Estado del Arte, se empleó como descriptores la motivación correlacionada con las palabras: educación, selección de carrera, profesional, universidad y educación superior. La selección de los artículos fue realizado en el mes de marzo de 2018, encontrando 294 artículos, de los cuales solo 18 estaban relacionados al contexto de esta investigación. Dichos artículos tienen informaciones referentes a la motivación, a los procesos motivacionales, a los factores motivacionales, a la autodeterminación y a la motivación escolar.

El análisis de los artículos seleccionados fue realizado mediante un Análisis de 
Contenido que, según Bardin (1977), es un conjunto de técnicas de análisis aplicadas en procesos sistemáticos y objetivos de descripción del contenido de los mensajes o textos que serán considerados objetos de estudios, a fin de inferir sobre los conocimientos relativos a los productos procesados, sea de forma cuantitativa o no. En el caso particular de esta investigación, se describen e interpretan las ideas principales que exponen los diferentes artículos sobre la motivación y su relación con la selección de carrera profesional, teniendo como resultado final la producción de un metatexto que expresa parte de los entendimientos de la comunidad científica sobre el tema.

En base a Bardin (1977), el análisis se realizó por medio de tres fases: a) la preexploración del material, permitiendo conocer el contexto y dejándose impregnar y orientar mediante una lectura fluctuante, tomando conocimiento de los aspectos relevantes de la motivación en los artículos; b) la selección de las unidades de análisis, consistió en tomar del corpus del texto extractos que permitieron dar respuesta a los cuestionamientos de la investigación; c) la categorización y sub-categorización, caracterizando las unidades de análisis en enunciados generales, según su grado de intimidad y de proximidad, pudiendo construir significados y elaboraciones. La categorización fue realizada de forma apriorista, tomando en cuenta el objetivo de la investigación.

\section{CONSIDERACIONES FINALES}

La motivación es un factor importante en la selección de la carrera profesional, como se ha podido apreciar, está estrechamente conectada a la motivación escolar y las motivaciones propias (valores, objetivos, necesidades, búsqueda de reconocimiento social, entre otras). A su vez relacionada con la cultura, la cual marcará las escalas de valores y necesidades de cada persona, haciendo que cada realidad estudiada sea diferente.

Figura 3. Diseño de Word Art con las palabras que más se repiten en los artículos analizados en el presente trabajo. 


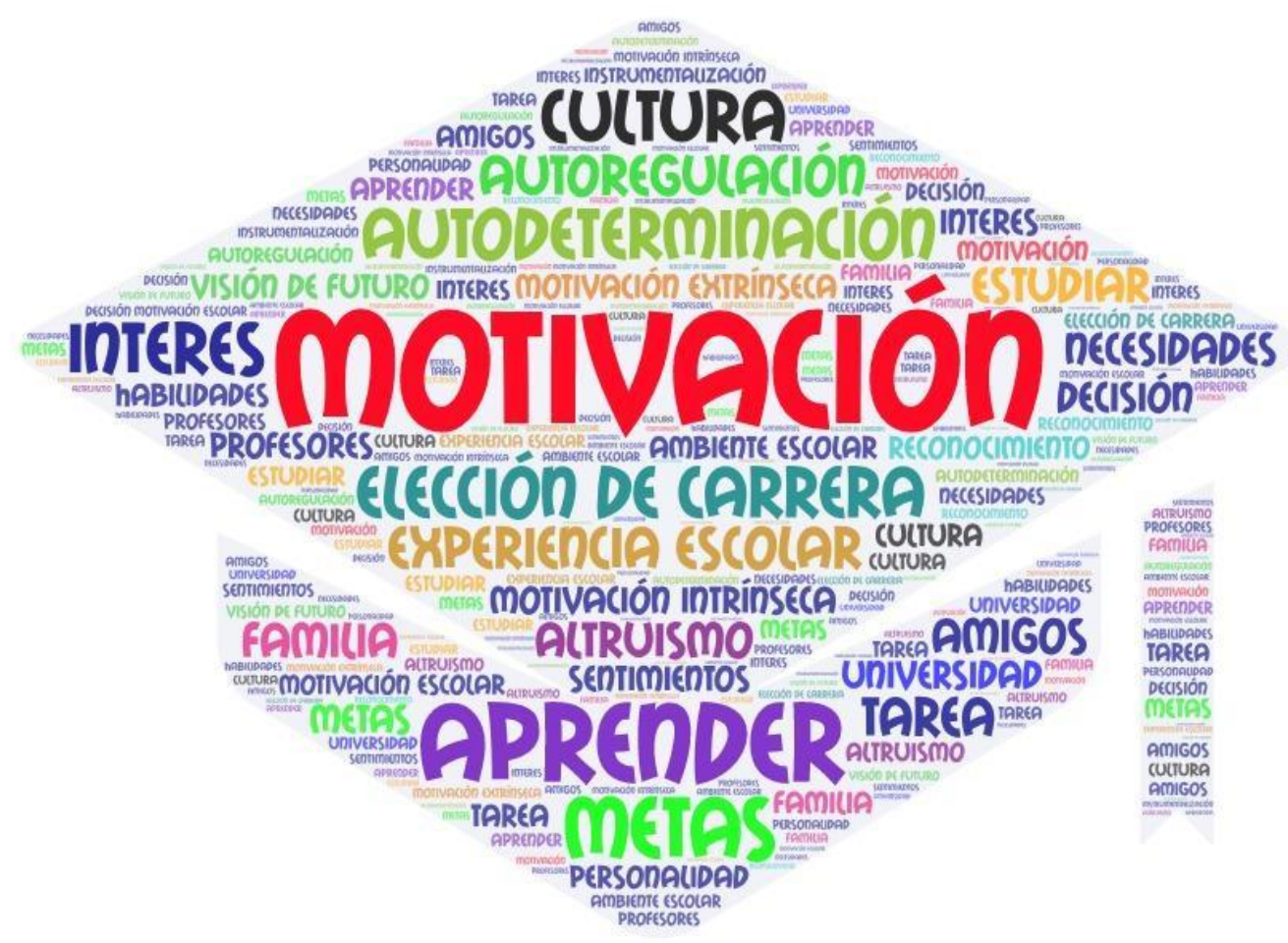

Fuente: https://wordart.com/create.

Los aspectos relacionados a la motivación en la toma de decisiones de una elección de carrera universitaria, han sido plasmados en la figura 3, mostrando las palabras claves en esta investigación. Teniendo como palabra central motivación, seguido por la motivación a aprender, lo cual dependerá de las metas personales, intereses, personalidad y los sentimientos o emociones envueltas en dicha elección.

También se refleja la autodeterminación y autoregulación, ambas teorías son de gran relevancia, pues permiten comprender mejor cómo ocurre la motivación intrínseca y extrínseca, los aspectos que actúan sobre la misma, como son la cultura, la familia, el contexto escolar, amigos, profesores, metas, habilidades, ímpetu por tener mejor instrumentalidad en algunas áreas o tipos de actividades. En otras palabras, comprender qué aspectos están presentes y como es la actitud hacia la actividad que nos hace movernos, actuar, en este caso particular, que hace tomar la decisión de estudiar determinada carrera profesional.

La motivación es entendida por la autodeterminación en dos grupos, como son la motivación intrínseca, aquella capaz de que una persona realice actividades simplemente por sentir placer, curiosidad o interés en obtener habilidades y la motivación extrínseca, por otro lado, se relaciona con las actividades que se hace por recibir algo a cambio, esto puede ser recompensas, por reconocimientos de otros o por querer cubrir una necesidad.

Sin embargo, estudios basados en la autorregulación identificaron diferentes niveles entre cada tipo de motivación, encontrando siete tipos de motivación, tres corresponden a la 
intrínseca y son para vivenciar estímulos, por realización, para saber. Las otras cuatro corresponden a la motivación extrínseca y corresponden a control externo, por introyección, por identificación y finalmente por integración. Cada una de ellas refleja una actitud diferente ante la actividad que va a realizar, teniendo preferencias por una motivación intrínseca, la cual está asociada, bajo un criterio de autonomía y autorregulación, actitudes de creatividad, persistencia y predisposición a la resolución de problemas.

En cuanto a la motivación escolar, existen diferentes enfoques o teorías que aportar ideas para lograr un mejor entendimiento de cómo influye la motivación tanto en la enseñanza como en el aprendizaje, además de las influencias culturales y sociales, que, de antemano, ya están envueltas en cada salón donde se desarrolla una clase.

La motivación en la selección de carrera profesional, al estar directamente asociada con la motivación para aprender, se nutre principalmente de un aprendizaje instrumental, es decir, aquel que logre ser considerado útil para la vida. Ya que esa necesidad de aprender o mejorar estará dada por esa visión de futuro de lo que se desea ser profesionalmente.

Por otra parte, la motivación en el contexto universitario depende del contexto sociocultural, del ambiente del aula, factores internos (personalidad) y los aspectos motivacionales intervinientes, como son los sentimientos, las percepciones, las necesidades, las metas y el desempeño académico, como puntos fundamentales en la determinación de un comportamiento motivado.

Se podría considerar que la motivación en la selección de carrera profesional es multifactorial, sin embargo, partiendo de la influencia que tiene la motivación escolar y específicamente la motivación en el aprendizaje, se hace relevante conocer la influencia motivacional del periodo escolar en la elección de carrera, por lo cual se sugiere en futuros estudios.

\section{Referencias}

AVENDAÑO, Cecilia; GONZALEZ, Rodrigo. Motives for entering to the careers of Pedagogy in students of first year at Universidad de Concepción. Estud. pedagóg. Valdivia, v.38, n.2, p.21-33, Dec. 2012. Disponible en: https://scielo.conicyt.cl/scielo.php?script=sci_arttext\&pid=S071807052012000200002\&lng=en\&nrm=iso. Acceso en: 04 mar. 2018.

BARDIN Laurence. Análise de conteúdo. Edições 70. 1977.

BELUCE, ANDREA CARVALHO; OLIVEIRA, KATYA LUCIANE DE. Escala de estratégias e motivação para aprendizagem em ambientes virtuais. Rev. Bras. Educ. Rio de Janeiro, $\quad$ v.21, n.66, p.593-610, sept. 2016. http://www.scielo.br/scielo.php?script=sci_arttext\&pid=S1413-

Disponible

en: 
24782016000300593\&lng=es\&nrm=iso. Accedido en: 04 mar. 2018.

BERNARDINO, Amanda de Oliveira et al. Motivação dos estudantes de enfermagem e sua influência no processo de ensino-aprendizagem. Texto contexto - enferm. Florianópolis, v.27, n.1, e1900016, $2018 . \quad$ Disponible en: http://www.scielo.br/scielo.php?script=sci_arttext\&pid=S0104-

07072018000100322\&lng=en\&nrm=iso. Acceso en: 04 y 20 mar. 2018.

BZUNECK, José Aloyseo; GUIMARAES, Sueli Édi Rufini. Estilos de professores na promoção da motivação intrínseca: reformulação e validação de instrumento. Psic.: Teor. e Pesq. Brasília, v.23, n.4, p.415-421, dic. $2007 . \quad$ Disponible en: http://www.scielo.br/scielo.php?script=sci_arttext\&pid=S010237722007000400007\&lng=es\&nrm=iso. Acceso en: 04 mar. 2018.

CANO, María Alicia. Motivación y Elección de Carrera. REMO. Mexico, v. 5, n. 13, p. 6-9, 2008 . Disponible en: <http://pepsic.bvsalud.org/pdf/remo/v5n13/v5n13a03.pdf > Acceso en: 10 abr. 2018.

ECCLES, Jacquelynne; WIGFIELD, Allan. In the Mind of the Actor: The Structure of Adolescents' Achievement Task Values and Expectancy-Related Beliefs. Personality and Social Psychology Bulletin n.21, v.3, p.215-225, 1995. Disponible en: http://hdl.handle.net/2027.42/69045. Acceso en: 20 jun. 2018.

FERREIRA, Norma Sandra de Almeida. As pesquisas denominadas "estado da arte". Educ. Soc. Campinas, v.23, n.79, p.257-272, Aug. 2002. Disponible en: http://www.scielo.br/scielo.php?script=sci_arttext\&pid=S0101 -

73302002000300013\&lng=en\&nrm=iso. Acceso en: 02 abr. 2018.

GARCIA GARDUNO, José María; ORGANISTA SANDOVAL, Javier. Motivación y expectativas para ingresar a la carrera de profesor de educación primaria: Un estudio de tres generaciones de estudiantes normalistas mexicanos de primer ingreso. REDIE. Ensenada, v.8, n.2, p.1-17, $2006 . \quad$ Disponible

en: http://www.scielo.org.mx/scielo.php?script=sci_arttext\&pid=S160740412006000200008\&lng=es\&nrm=iso. Acceso en: 04 mar. 2018

GÓES, N., ALLIPRANDINI P. Análise das estrategias de aprendizagens cognitivas, autorregulatórias e comportamentais utilizadas por alunos de um curso de pedagogia ofertado a distância. X ANPED SUL. 2014. Disponible en: http://xanpedsul.faed.udesc.br/arq_pdf/330-0.pdf. Acceso en: 28 jun. 2018.

MARTINEZ-HERNANDEZ, Ariadna Crisantema; VALDERRAMA-JUAREZ, Lorena Elizabeth. Motivación para Estudiar en Jóvenes de Nivel Medio Superior. Nova scientia. León, v.3, n.5, p.164-178, 2011. Disponible http://www.scielo.org.mx/scielo.php?script=sci_arttext\&pid=S200707052011000100009\&lng=es\&nrm=iso. Acceso en: 04 de mar. 2018.

MONTALVO-AGUILAR, Michell et al . Desempeño académico en el primer año de medicina: relación con intereses vocacionales, actitud ante el estudio y fuerza de motivación. FEM (Ed. impresa), Barcelona, v.16, n.2, p.111-117, jun. 2013. Disponible en: $\quad$ http://scielo.isciii.es/scielo.php?script=sci_arttext\&pid=S201498322013000200010\&lng=es\&nrm=iso. Acceso en: 10 abr. 2018. 
PABLO-LERCHUNDI, Iciar; NÚÑEZ-del-Río, María-Cristina; GONZÁLEZ-TIRADOS, Rosa-María. Career choice in engineering students: its relationship with motivation, satisfaction and the development of professional plans. Anales de Psicología. Murcia, v.31, n.1, p.268-279, enero-abril, 2015. Disponible en: http://revistas.um.es/analesps/article/view/analesps.31.1.159161/169801. Acceso en: 10 abr. 2018.

PAIVA, Mirella Lopez Martini Fernandes; BORUCHOVITCH, Evely. Orientações motivacionais, crenças educacionais e desempenho escolar de estudantes do ensino fundamental. Psicol. estud., Maringá , v. 15, n. 2, p. 381-389, June 2010 . Disponible en: http://www.scielo.br/scielo.php?script=sci_arttext\&pid=S1413-

73722010000200017\&lng=en\&nrm=iso. Acceso en: 04 mar. 2018

PEREIRA, Alan Antunes; NOGUEIRA, Anelise de Barros Leite; CABETTE, Regina Elaine Santos. Motivação em universitários: análises de teses e dissertações entre 2000 e 2011. Psicol. Esc. Educ. Maringá, v.21, n.2, p.323-331, Aug. 2017. Disponible en: http://www.scielo.br/scielo.php?script=sci_arttext\&pid=S1413-

85572017000200323\&lng=en\&nrm=iso. Acceso en: 04 mar. 2018.

PINTRICH Paul. A Motivational Science Perspective on the Role of Student Motivation in Learning and Teaching Contexts. Journal of Educational Psychology. v.95, n.4, p.667-786, jun. 2003.2 Disponible en: https://ueeval.ucr.edu/teaching_practices_inventory/Pintrich_2003.pdf. Acceso en: 15 jun. 2018

PINTRICH Paul; DE GROOT Elizabeth. Motivational and Self-Regulated Learning Components of Classroom Academic Performance. Journal of Educational Psychology. v.82, n.1, p.33-40, sep. 1990. Disponible en: http://rhartshorne.com/fall-2012/eme6507$\mathrm{rh} /$ cdisturco/eme6507-eportfolio/documents/pintrich\%20and\%20degroodt\%201990.pdf.

Acceso en: 15 jun. 2018

RAMÍREZ, Pedro; CORTES, Patricia. Motivación y Rendimiento en una carrera del área de Química de la Universidad Católica del Norte - Chile. Psicoperspectivas. v. 2, p. 129-155, 2008.

Disponible

en:

http://www.psicoperspectivas.cl/index.php/psicoperspectivas/article/viewFile/7/7. Acceso en: 02 mar. 2018.

RUFINI, Sueli Édi; BZUNECK, José Aloyseo; OLIVEIRA, Katya Luciane de. A qualidade da motivação em estudantes do ensino fundamental. Paidéia (Ribeirão Preto). Ribeirão Preto, v.22, n.51, p.53-62, abr. 2012. Disponible en: http://www.scielo.br/scielo.php?script=sci_arttext\&pid=S0103-

863X2012000100007\&lng=es\&nrm=iso. Acesso en: 01 oct. 2018.

REEVE Jonhmarshall, DECI Edward, RYAN Richard. Self-determination theory: A dialectical framework for understanding sociocultural influences on student motivation. 2004. Disponible en: https://www.researchgate.net/publication/309563565. Acceso en: 20 jun. 2018.

SAID-HUNG, Elias; GRATACOS, Gloria; COBOS, Jorge Valencia. Factores que influyen en la elección de las carreras de pedagogía en Colombia. Educ. Pesqui. São Paulo, v.43, n.1, p.31-48, Mar. 2017. Disponible en: http://www.scielo.br/pdf/ep/v43n1/1517- 
9702-ep-43-1-0031.pdf. Acceso en: 04 mar. 2018.

SKATOVA, Anya; FERGUSON, Eamonn. Why do differente people choose different university degrees? Motivation and the choice of degree. Frontiers in Psychology. v.5, p.115, nov. 2014. Disponible en: https://www.researchgate.net/publication/266794683_Why_do_different_people_choose_diff erent_university_degrees_Motivation_and_the_choice_of_degree?enrichId=rgreqd0284b802cfc6eefadc7b451d370c090-

XXX\&enrichSource=Y292ZXJQYWd1OzI2Njc5NDY4MztBUzoxNjMwNjQ2OTk2OTUxM DRAMTQxNTg4OTA2MjA3NA\%3D\%3D\&el=1_x_2\&_esc=publicationCoverPdf. Acceso en: 10 abr. 2018.

STEINMANN, Andrea; BOSCH, Beatriz; AIASSA, Delia. Motivación y expectativas de los estudantes por aprender ciencias en la universidad: un estudio exploratorio. Revista Mexicana de Investigación Educativa. México, v.18, n.57, p.585-598, 2013. Disponible en: http://www.redalyc.org/pdf/140/14025774012.pdf. Acceso en: 02 mar. 2018.

TONELOTTO, Joseane. Aprender como aprender: estratégias metacognitivas como ferramentas do proceso de ensino-aprendizaje. Associacão Brasileira de Mantenedoras do Ensino Superior Blog. 2012. Disponible en: HTTP://blog.abmes.org.br/?p=3735. Acceso en: 29 jun. 2018.

VALENZUELA Jorge; MUÑOZ, Carla; SILVA-PEÑA, Hich; GÓMEZ, Viviana; PRECHT, Andrea. Motivación escolar: claves para la formación motivacional de futuros docentes. Estudios pedagógicos. Chile, v.41, n.1, p.351-361. 2015. Disponible en: http://www.redalyc.org/articulo.oa?id=173541114021. Acceso en: 02 mar. 2018.

WATT, H.M.G., \& RICHARDSON, P.W. Motivational factors influencing teaching as a career choice: Development and validation of the FIT-Choice scale. Journal of Experimental Education. n.75, p.167-202, 2007. Disponible en: https://www.researchgate.net/profile/Helen_Watt/publication/238047395_Motivational_Facto rs_Influencing_Teaching_as_a_Career_Choice_Development_and_Validation_of_the_FITChoice_Scale/links/00b49520ebd24add7e000000/Motivational-Factors-InfluencingTeaching-as-a-Career-Choice-Development-and-Validation-of-the-FIT-ChoiceScale.pdf?origin=publication_detail. Acceso en: 15 jun. 2018. 